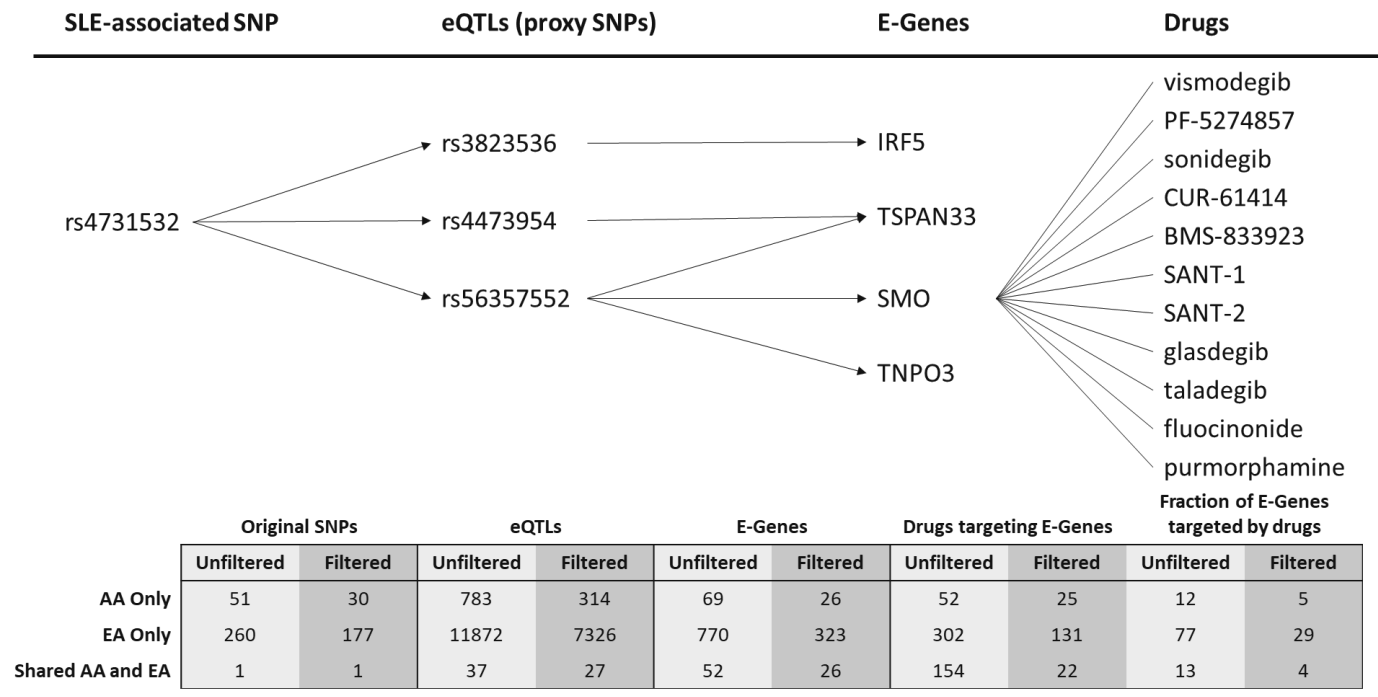

Abstract BD-01 Figure 1 Unpacking an SLE-associated SNP

with an increased burden of co-morbidities compared to European-American (EA) populations. Genome-wide association studies (GWAS) have linked many single nucleotide polymorphisms (SNPs) to SLE. Recently, Langefeld (2017) conducted a large-scale transancestral association study of SLE to identify ancestry-dependent and independent contributions to SLE risk. We extend these findings to include a transancestral analysis linking SLE-associated SNPs to candidate-causal E-Genes specific to AA and EA populations and differential gene expression in these populations with the goal of matching genetic and genomic disease characteristics with available treatments unique to each ancestral group.

Methods SNPs proxy to SLE-associated SNPs were compared with known expression quantitative trait loci (eQTLs) contained in the GTEx (version 6) database. E-QTLs and their associated E-Genes were divided by ancestry and compared to differentially expressed (DE) genes from multiple SLE gene expression datasets. For both ancestral groups, E-Gene lists were examined for the significant enrichment of BIG-C categories and IPA (Qiagen) Canonical Pathways to predict novel upstream regulators (UPRs). For visualization and clustering analysis, STRING-generated networks of DE E-Genes were imported into Cytoscape (version 3.6.1) and partitioned with the community clustering (GLay) algorithm via the clusterMaker2 (version 1.2.1) plugin. Finally, drug candidates targeting E-Genes, DE genes and UPRs were identified using CLUE, REST, API, IPA and STITCH (version 5.0; http://stitch.embl. de). The process of unpacking an SLE-associated SNP is shown in figure 1.

Results E-QTL and DE gene queries of GTEx were combined and newly predicted E-Genes were pooled by ancestry. Here, we identify 52 SNPs with eQTLs unique to AA ancestry, 260 SNPs unique to EA ancestry and 1 SNP shared between ancestries. Together, these SNPs identified a total of 891 distinct EGenes associated with both ancestral groups. In studies comparing E-Genes to SLE DE data sets, 516 EA E-Genes were differential expressed compared to 48 AA E-Genes. Comparison with various drug candidate databases resulted in the identification of 12 drugs targeting genes specific for AA, 77 drugs specific for EA genes and 13 shared between EA and AA. Predicted EA-specific drugs include hydroxychloroquine and drugs-in-development targeting CD40LG, CXCR1 and CXCR2 whereas AA-specific drugs include HDAC inhibitors, retinoids, and drugs targeting IRAK4 and CTLA4. Drugs targeting EGenes/pathways shared by EA and AA include ibrutinib, ruxolitinib and ustekinumab.

Conclusions The ancestral SNP-associated E-Genes and gene expression profiles outlined here illustrate fundamental differences in lupus molecular pathways between AA and EA. Our results indicate that unique sets of drugs may be particularly effective at treating lupus within each ancestral group.

Acknowledgments Financial support for this research was provided by RILITE Research Institute.

\section{BD-02 BLOCKADE OF THE MECHANISTIC TARGET OF RAPAMYCIN ELICITS RAPID AND LASTING IMPROVEMENT OF DISEASE ACTIVITY THROUGH RESTRAINING PRO-INFLAMMATORY T CELL LINEAGE SPECIFICATION IN PATIENTS WITH ACTIVE SLE}

Zhi-Wei Lai, Ryan Kelly, Thomas Winans, Ivan Marchena, Ashwini Shadakshari, Julie Yu, Maha Dawood, Ricardo Garcia, Hajra Tily, Lisa Francis, Stephen V Faraone, Paul E Phillips, Andras Perl*. Division of Rheumatology, Departments of Medicine, Microbiology and Immunology, and Biochemistry and Molecular Biology, State University of New York, Upstate Medical University, College of Medicine, Syracuse, New York

\subsection{6/lupus-2018-Ism.26}

Background Systemic lupus erythematosus (SLE) patients exhibit T-cell dysfunction that has been attributed to mechanistic target of rapamycin activation. Therefore, safety, tolerance, and efficacy of rapamycin were examined in a prospective biomarker-driven open-label clinical trial.

Methods 40 patients having active disease and unresponsive or intolerant to conventional medications were enrolled. Sirolimus was started at $2 \mathrm{mg} /$ day with dosage adjusted to tolerance and 6-15 ng/ml trough levels. Disease activity was evaluated by BILAG, SLEDAI, and prednisone use over 12 months. Blood samples of 56 matched healthy subjects were obtained as controls for immunometabolic outcomes monitored at each visit. Results 11 patients dropped out, 9 for non-compliance and 2 for intolerance. Among safety outcomes, liver function and lymphocyte counts were unchanged. While HDL-cholesterol, neutrophil counts and haemoglobin were moderately reduced, 


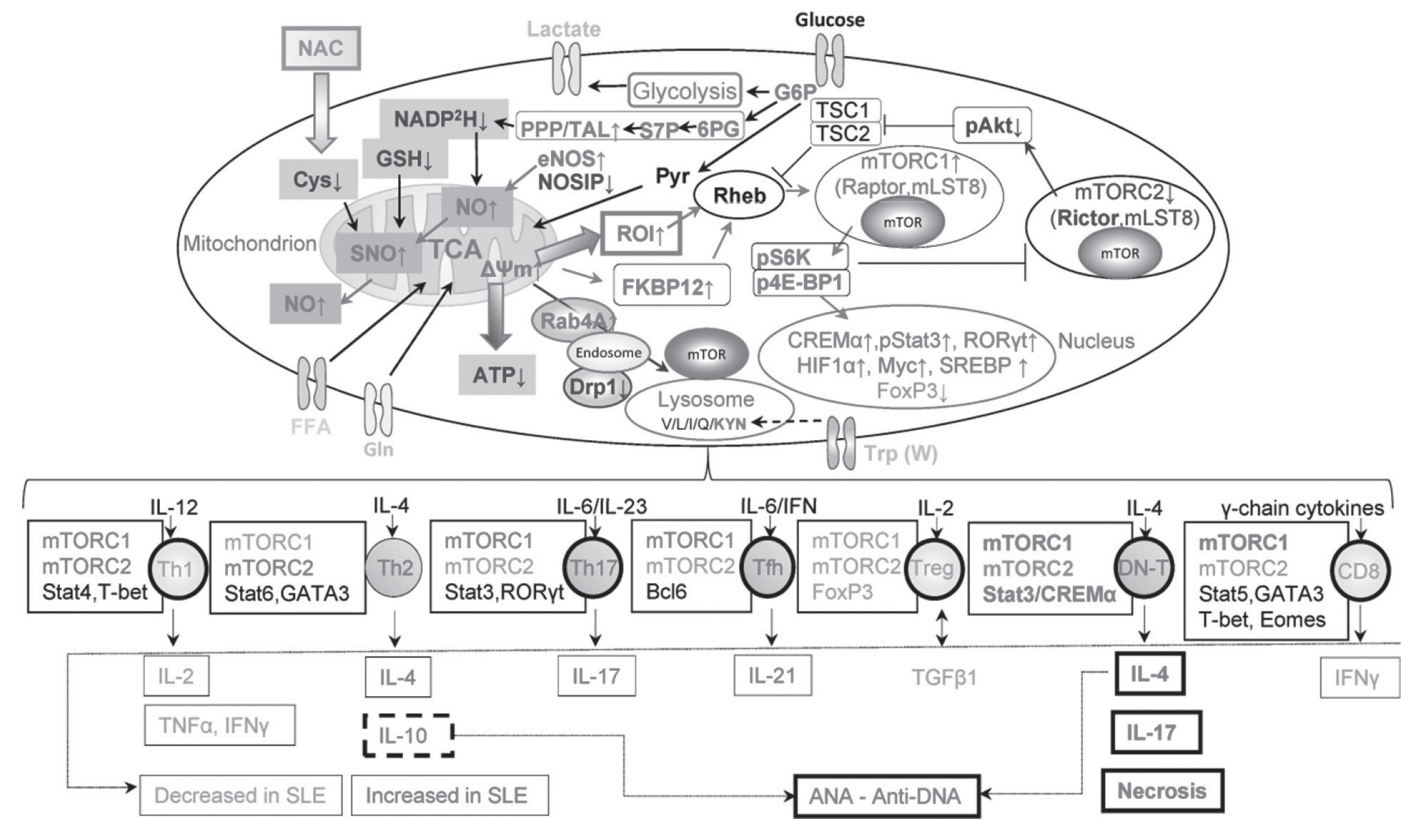

Abstract BD-02 Figure 1 Metabolic control of pro-inflammatory T-cell lineage specification in SLE. Schematic molecular order of pathways upstream and downstream of activation of the mechanistic target of rapamycin (mTOR) in SLE. mTOR is activated on the surface of lysosomes in a state of amino acid sufficiency (V/L//Q/Kyn). ${ }^{1}$ Oxidative stress, in particular cysteine oxidation, also activates mTORC1 through association with Rheb. ${ }^{2}$ Given the results of our randomized double-blind placebo-controlled clinical trial showing that therapeutically effective reversal of GSH depletion by NAC blocks mTORC1 in vivo, ${ }^{3}$ GSH depletion will be considered the primary metabolic checkpoint of pro-inflammatory T-cell lineage specification in SLE. The depletion of GSH will be mechanistically connected to the depletion of cysteine (Cys) and NADPH and to the accumulation of kynurenine (Kyn) which have been uncovered by comprehensive metabolome studies of PBL from SLE and healthy subjects matched for age, gender, and ethnicity and processed in parallel. ${ }^{4}$ Blockade of mTOR with rapamycin reverses the depletion of effector-memory CD8 T cells and Tregs and the expansion of pro-inflammatory CD4 CD8 ${ }^{-}$double-negative T cells in patients with active SLE in vivo ${ }^{5}$. Red and blue arrows reflect direction of changes in SLE.

all changes occurred within a range considered safe. Platelet counts were slightly elevated over 12 months. As primary clinical efficacy endpoint, SLEDAI and BILAG disease activity scores were reduced over 12 months in $16 / 29$ patients $(55 \%)$. 19/29 patients (65.5\%) met criteria for SLE Responder Index (SRI). Arthritis, rash, pyuria, and hypocomplementemia improved among SLEDAI components, while cardiopulmonary, musculoskeletal, mucocutaneous, and vasculitis BILAG organdomain scores also declined. Prednisone use diminished from $24.3 \pm 4.7 \mathrm{mg} /$ day to $7.2 \pm 2.3 \mathrm{mg} / \mathrm{day} \quad(\mathrm{p}<0.0009)$. Sirolimus expanded $\mathrm{CD}^{+} \mathrm{CD} 25^{+} \mathrm{FoxP}^{+}$Tregs and $\mathrm{CD} 8^{+}$memory $\mathrm{T}$ cells and inhibited IL-4 and IL-17 production by $\mathrm{CD}^{+}{ }^{+}$and CD4 ${ }^{-}{ }^{-} 8^{-}$double-negative $\mathrm{T}$ cells after 12 months. $\mathrm{CD} 8^{+}$ memory $\mathrm{T}$ cells were selectively expanded in SRI-responders.

Conclusions Sirolimus elicits rapid, progressive, and sustained improvement of disease activity by correcting pro-inflammatory T-cell lineage specification in patients with active SLE.

Acknowledgements This work was supported in part by an Investigator-Initiated Research Grant P0468 × 1-4470/ WS1234172 from Pfizer and grants AI 048079, AI 072648, and AI 122176 from the National Institutes of Health and the Central New York Community Foundation.

Trial registration Prospective Study of Rapamycin for the Treatment of SLE; ClinicalTrials.gov Identifier: NCT00779194. Treatment trial of SLE with N-acetylcysteine; ClinicalTrials.gov identifier: NCT00775476.

\section{REFERENCES}

1. Perl A. Mechanistic target of rapamycin pathway activation in rheumatic diseases. Nat. Rev. Rheumatol. 2016;12:169-82.
2. Yoshida $\mathrm{S}$, et al. Redox regulates mammalian Target of Rapamycin Complex 1 (mTORC1) activity by modulating the TSC1/TSC2-Rheb GTPase pathway. J. Biol. Chem 2011;286:32651-60.

3. Lai Z-W, et al. N-acetylcysteine reduces disease activity by blocking mTOR in T cells of lupus patients. Arthritis Rheum 2012;64:2937-46.

4. Perl $\mathrm{A}$, et al. Comprehensive metabolome analyses reveal $\mathrm{N}$-acetylcysteine-responsive accumulation of kynurenine in systemic lupus erythematosus: Implications for activation of the mechanistic target of rapamycin. Metabolomics 2015;11:115774.

5. Lai $Z$, et al. Sirolimus in patients with clinically active systemic lupus erythematosus resistant to, or intolerant of, conventional medications: A single-arm, openlabel, phase 1/2 trial. Lancet 2018;391:1186-96.

\section{BD-03 ADHERENCE TO ANTIMALARIALS AND RISK OF TYPE 2 DIABETES MELLITUS IN PATIENTS WITH SYSTEMIC LUPUS ERYTHEMATOSUS: A POPULATION-BASED COHORT STUDY}

1J Antonio Avina-Zubieta*, ${ }^{2}$ Hans D Haag, ${ }^{1}$ Eric C Sayre, ${ }^{1}$ John M Esdaile, ${ }^{1,2}$ Mary A De Vera. 'Arthritis Research Canada, The University of British Columbia; ${ }^{2}$ Faculty of Pharmaceutical Sciences, The University of British Columbia, Canada

\subsection{6/lupus-2018-Ism.27}

Background Aside from their effect on disease activity in systemic lupus erythematosus (SLE) antimalarials have been shown additional benefits such as reducing the risk of diabetes. However, a recent systematic review reported sub-optimal adherence to antimalarials with rates as low as $25 \%$. As medication adherence mediates patient outcomes, our objective was to evaluate the association between adherence to antimalarials 